\title{
On the classification of solutions of cosmic strings equation
}

\author{
Weiwei $\mathrm{Ao}^{1} \cdot$ Wen Yang $^{2}$ (D)
}

Received: 14 October 2018 / Accepted: 13 April 2019 / Published online: 13 May 2019

(c) Fondazione Annali di Matematica Pura ed Applicata and Springer-Verlag GmbH Germany, part of Springer Nature 2019

\section{Abstract}

In this paper, we prove some Liouville-type theorems for weak stable or finite Morse index solutions to the following equation:

$$
\Delta u+e^{u}+|x|^{\alpha} e^{\beta u}=0 \text { in } \mathbb{R}^{N},
$$

which arises from the study of selfgravitating cosmic strings for a massive $W$-boson model coupled with Einstein's equation.

Mathematics Subject Classification 35J15 $\cdot 35 \mathrm{~J} 60 \cdot 35 \mathrm{~J} 61$

\section{Introduction}

In this short paper, we study and classify the stable or finite Morse index weak solutions of the semi-linear partial differential equation

$$
\Delta u+e^{u}+|x|^{\alpha} e^{\beta u}=0 \text { in } \mathbb{R}^{N}, \quad N \geq 2 .
$$

The motivation to study Eq. (1.1) on this type comes from the selfgravitating strings for a massive $W$-boson model coupled to Einstein theory in account of gravitational effects. More precisely, for the superconducting cosmic string model (see [1]), Yang in [33] introduced a set of ansatz so that the string configuration of the original model satisfies a system of Bogomolyni-type (selfdual) first-order equation coupled with Einstein's equation, based on this governing string's system, by making full use of the boundary condition, we find that a term which involves the conformal factor and the strength of the $W$-boson field is a constant, and it reduces the system to a single equation, which is (1.1), where $\frac{\alpha}{2} \in \mathbb{N}$ represents the

$凶 \quad$ Wen Yang

wyang@wipm.ac.cn

Weiwei Ao

wwao@whu.edu.cn

1 School of Mathematics and Statistics, Wuhan University, Wuhan 430072, People's Republic of China

2 Wuhan Institute of Physics and Mathematics, Chinese Academy of Sciences, P.O. Box 71010, Wuhan 430071, People's Republic of China 
string's multiplicity and $\beta>0$ is a physical parameter, see $[1,20,32,33]$ for the derivation of (1.1) and the physical background from the corresponding Einstein-Weinberg-Salam theory.

For Eq. (1.1), some answer on the solvability of (1.1) in the context of radially symmetric solution of dimension 2 has been investigated by Poliakovsky and Tarantello recently, see [20]. When $\alpha=0,(1.1)$ is related to the description of turbulence flows first proposed by Onsager [19], in the case in which all vortices have the same orientation. We refer the readers to $[12,17-19,23,24]$ and references therein for more background on it. It is also interesting to point out that, when $\alpha=2 \beta-2$, problem (1.1) shares many properties with either the Liouville equation or the "singular" Liouville equation. Indeed, Chen, Guo and Spirn [5] show that, if $\alpha>-2$ and $u$ is a solution to (1.1), then

(i) $\int_{\mathbb{R}^{2}}\left(e^{u}+|x|^{\alpha} e^{\beta u}\right) \mathrm{d} x=\frac{4 \pi(\alpha+2)}{\beta}$,

(ii) $u_{\lambda}(x)=u(\lambda x)+2 \log \lambda, \hat{u}(x)=u\left(\frac{x}{|x|^{2}}\right)+4 \log \frac{1}{|x|}$ are also solutions for (1.1).

In this special case, the radial solutions can be expressed in terms of some elliptic integrals, and we can only get the explicit formula when $\alpha=2$, that is

$$
u(x)=\log \left(\frac{\Lambda^{2}}{1+\frac{1}{4} \Lambda^{2}|x|^{2}+\frac{5}{64} \Lambda^{4}|x|^{4}}\right), \quad \Lambda>0,
$$

see (2.15) in [20]. While for non-radial solutions, until now we still have no results on that. For the case $\alpha \neq 2 \beta-2$, then the existence result is very subtle, even for the radially symmetric solutions, and it may depend on the choices of $\alpha, \beta$ and the total integration of the nonlinear term, see the results in [5,20]. Apart from the existence result of (1.1), Tarantello in [26] studied the blow up behavior of the bubbling solution of (1.1) in $\mathbb{R}^{2}$. For the related work, we refer the readers to $[2-4,15,21,22,27]$ and references therein.

The purpose of this paper is to consider the stable solutions of this problem, which can be seen as the continuation of the work of Dancer-Farina [7] on Liouville equation and Wang-Ye [28] on Hénon-type elliptic equation. There is an extensive literature concerning the stable solutions of the second-order or fourth-order elliptic equations. By the Moser's iteration method, Farina [14] completely classified all finite Morse index classical solutions of the Lane-Emden equation

$$
\Delta u+|u|^{p-1} u=0 \text { in } \mathbb{R}^{N},
$$

provided $p \in\left(1, p_{J L}\right)$ with $p_{J L}$ given by

$$
p_{J L}= \begin{cases}+\infty, & \text { if } N \leq 10, \\ \frac{(N-2)^{2}-4 N+8 \sqrt{N-1}}{(N-2)(N-10)}, & \text { if } N \geq 11 .\end{cases}
$$

Based on the method and techniques developed in [14], Dancer-Farina undertake the classification of solutions of the equation

$$
\Delta u+e^{u}=0 \text { in } \mathbb{R}^{N},
$$

and obtained the above equation does not admit any solution stable for $2 \leq N \leq 9$ or with finite Morse index for $3 \leq N \leq 9$. By a completely different approach, Dávila, Doupaigne, Wang and Wei [11] classify the stable and finite Morse index solutions to the fourth-order supercritical problem in full exponent range on the basis of monotonicity formula. For the fourth-order problem with exponential nonlinearity, unlike the corresponding second-order problem, no Liouville-type theorem holds, unless we get more information on the asymptotic behavior of solutions at infinity, see [13]. For the nonautonomous case, Wang and Ye 
obtained a similar Liouville-type results both for the Hénon-type Lane-Emden equation and Liouville equation, in the spirit of Farina [14] for Lane-Emden equation and Dancer-Farina for Liouville equation, respectively. For the other related works, we refer the readers to $[6,8-10,16,30,31]$ and references therein.

As addressed before, Eq. (1.1) shares the scale invariant property if $\alpha=2 \beta-2$, and we can obtain the monotonicity formula for (1.1):

$$
\begin{aligned}
E(u, 0, \lambda)= & \lambda^{2-N} \int_{B(0, \lambda)}\left(\frac{1}{2}|\nabla u|^{2}-e^{u}-\frac{2}{2+\alpha}|x|^{\alpha} e^{\frac{1}{2}(2+\alpha) u}\right) \mathrm{d} x \\
& +2 \lambda^{1-N} \int_{\partial B(0, \lambda)}(u(x)+2 \log \lambda) \mathrm{d} \sigma
\end{aligned}
$$

and one can easily check that

$$
\frac{\mathrm{d}}{\mathrm{d} \lambda} E(u, 0, \lambda)=\lambda^{1-N} \int_{\partial B(0, \lambda)}\left(\partial_{r} u+\frac{2}{r}\right)^{2} \mathrm{~d} \sigma \geq 0 .
$$

Using this monotonicity formula (1.2), one may be able to obtain some nonexistence results for the stable solutions of Eq. (1.1) when $\alpha=2 \beta-2$, in the spirit of the work [11,29]. Instead of using the monotonicity formula, we shall apply the modified techniques in [28], to classify the stable or finite Morse index solutions of the problem (1.1) in more general situation (including the scale invariant case).

To state our results, let us give the precise definition of weak solution and stable or finite Morse index solutions in the following

Definition We say that $u$ is a weak solution of $-\Delta u=f(x, u)$ in domain $\Omega \subset \mathbb{R}^{N}$ (bounded or not), if $u \in H_{\text {loc }}^{1}(\Omega)$ verifies the equation with $f(x, u) \in L_{\text {loc }}^{1}(\Omega)$ and

$$
\int_{\Omega}(\nabla u \nabla \phi-f(x, u) \phi)=0, \quad \forall \phi \in C_{c}^{1}(\Omega)
$$

where $C_{c}^{k}(\Omega)$ denotes the set of $C^{k}$ functions with compact support in $\Omega$, we say this weak solution $u$

- is stable if $\partial_{u} f(x, u) \in L_{\mathrm{loc}}^{1}(\Omega)$ and

$$
Q_{u}(\phi):=\int_{\Omega}\left[|\nabla \phi|^{2}-\partial_{u} f(x, u) \phi^{2}\right] \geq 0, \quad \forall \phi \in C_{c}^{1}(\Omega),
$$

- has Morse index equal to $K \geq 1$ if $K$ is the maximal dimension of a subspace $X_{K}$ of $C_{c}^{1}(\Omega)$ such that $Q_{u}(\phi)<0$ for any $\phi \in X_{K} \backslash\{0\}$,

- is stable outside a compact set $\mathcal{K} \subset \Omega$ if $Q_{u}(\phi) \geq 0$ for any $\phi \in C_{c}^{1}(\Omega \backslash \mathcal{K})$.

We should remark that any finite Morse index solution $u$ is stable outside a compact set $\mathcal{K} \subset \Omega$. Indeed, for any finite Morse index solution, there exists a number $\ell$ such that for the span of $\left\{\phi_{1}, \ldots, \phi_{\ell}\right\}, Q_{u}(\phi)<0$ for any $\phi \in X \backslash\{0\}$. So $Q_{u}(\phi) \geq 0$ for all $\phi \in C_{c}^{1}(\Omega \backslash \mathcal{K})$, where $\mathcal{K}=\bigcup_{j=1}^{\ell} \operatorname{supp}\left(\phi_{j}\right)$.

The main results of this paper are the following:

Theorem 1.1 Let $\alpha>-2, \beta>0$ and $\Omega=\mathbb{R}^{N}$. For $2 \leq N<2+\frac{4(\alpha+2)}{\max \{1, \beta\}}$, there is no weak stable solution of (1.1).

Remark 1 When $\alpha=0, \beta=1$, from Theorem 1.1 we get that there is no weak stable solution of the Liouville equation provided $2 \leq N \leq 9$, which coincides with the result obtained in [7]. 
Remark 2 Based on Theorem 1.1, we can show that all the radially symmetric solutions obtained in [20] are unstable.

Theorem 1.2 Let $\alpha>-2, \beta>0$ and $\Omega=\mathbb{R}^{N}$. If $\alpha+2 \geq 2 \beta$ and $2<N<2+8 \min \left\{\beta, \frac{1}{\beta}\right\}$, there is no weak solution to (1.1) which is stable outside a compact set.

Remark 3 From Theorem 1.2 and the fact that any finite Morse index solution is stable outside a compact set $\mathcal{K} \subset \Omega$, we directly obtain that under the same assumption of Theorem 1.2, there is no weak finite Morse index solution to (1.1).

Let us close this introduction by making some comments that the techniques used in this paper can also be used to treat the following equation

$$
\Delta u+|u|^{p-1} u+|x|^{\alpha}|u|^{q-1} u=0,
$$

and it is also interesting to notice that above equation also enjoys the scale invariant property provided $\alpha=\frac{2 q-2 p}{p-1}$.

In next section, we shall provide the proof of our main results, Theorems 1.1 and 1.2.

\section{Proof of Theorem 1.1 and Theorem 1.2}

At the beginning of this section, we shall show that $\alpha>-2$ is necessary, due to the following nonexistence result:

Lemma 2.1 For $\alpha \leq-2$, Eq. (1.1) with $\beta>0$ admits no weak solutions for any domain $\Omega \subset \mathbb{R}^{N}$ which containing 0 .

Proof Suppose $u$ is a weak solution of (1.1) with $0 \in \Omega$. Let $B(0, R) \subset \Omega$ and $v$ be the average of $u$ over spheres centered at 0 . By Jensens inequality,

$$
-\Delta v=\overline{e^{u}}+r^{\alpha} \overline{e^{\beta u}} \geq e^{v}+r^{\alpha} e^{\beta v} .
$$

By the equation satisfied by $v$,

$$
-r^{N-1} \omega_{N} v^{\prime}(r)=-\int_{\partial B(0, r)} \frac{\partial v}{\partial v} \mathrm{~d} \sigma=-\int_{B(0, r)} \Delta v \mathrm{~d} x=\int_{B(0, r)}\left[e^{u}+r^{\alpha} e^{\beta u}\right] \mathrm{d} x>0,
$$

and it gives that $v^{\prime}(r)<0$ for $r \in(0, R)$. Here $\omega_{N}$ denotes the surface area of $N$-dimensional unit ball.

Integrating (2.1) from $r_{1}$ to $r$, we have

$$
-r^{N-1} v^{\prime}(r)+r_{1}^{N-1} v^{\prime}\left(r_{1}\right) \geq \int_{r_{1}}^{r} s^{N-1}\left[e^{v(s)}+|s|^{\alpha} e^{\beta v(s)}\right] \mathrm{d} s .
$$

Since $e^{v}, r^{\alpha} e^{\beta v} \in L_{\text {loc }}^{1}(\Omega)$, we can send $r_{1}$ to 0 in the integration. Together with the fact that $v^{\prime}(r)$ is negative for $r \in(0, R)$, one has

$$
-r^{N-1} v^{\prime}(r) \geq \int_{0}^{r} s^{N-1}\left[e^{v(s)}+|s|^{\alpha} e^{\beta v(s)}\right] \mathrm{d} s>C e^{\beta v(r)} r^{N+\alpha},
$$

and it implies that

$$
e^{-\beta v(r)} \geq C \int_{r_{1}}^{r} s^{1+\alpha} \mathrm{d} s \rightarrow \infty
$$

as $r_{1} \rightarrow 0$ if $\alpha \leq-2$. This is a contradiction. So the lemma is proved. 
Next we state the following estimate in the spirit of [28], which is crucial in the proof of the main theorems.

Proposition 2.1 Let $\Omega$ be a domain in $\mathbb{R}^{N}, N \geq 2$. Let $u$ be a weak and stable solution of (1.1) with $\alpha>-2, \beta>0$. Then for any integer $m \geq 1+\frac{4}{\max \{\beta, 1\}}$ and any $\gamma \in(0, \min \{2,2 \beta\})$, there exists $C>0$ depending on $m, \alpha, \beta$ and $\gamma$ such that

$$
\int_{\Omega}\left[e^{(2 \gamma+1) u}+|x|^{\alpha} e^{(\beta+2 \gamma) u}\right] \psi^{2 m} d x \leq C \int_{\Omega}|x|^{-\frac{2 \alpha \gamma}{\beta}}\left(|\nabla \psi|^{2}+|\psi||\Delta \psi|\right)^{\frac{\beta+2 \gamma}{\beta}} \mathrm{d} x
$$

for all functions $\psi \in C_{0}^{\infty}(\Omega)$ with $\|\psi\|_{\infty} \leq 1$.

Proof Let $\gamma>0, k \in \mathbb{N}$ and $k \geq \gamma^{-1}$. Define three functions $\tau_{k}(t), \eta_{k}(t)$ and $\xi_{k}(t)$ such that they satisfy $\eta_{k}^{\prime}(t)=\left[\tau_{k}^{\prime}(t)\right]^{2}$ and $\xi_{k}^{\prime}(t)=\eta_{k}(t)$ where

$$
\tau_{k}(t)=\left\{\begin{array}{ll}
e^{\gamma t}, & t \leq k, \\
\frac{e^{\gamma k}}{k} t, & t \geq k,
\end{array} \quad \eta_{k}(t)= \begin{cases}\frac{\gamma}{2} e^{2 \gamma t}, & t \leq k, \\
\frac{e^{2 \gamma k}}{k^{2}}(t-k)+\frac{\gamma}{2} e^{2 \gamma k}, & t \geq k,\end{cases}\right.
$$

and

$$
\xi_{k}(t)= \begin{cases}\frac{e^{2 \gamma t}}{4}, & t \leq k, \\ \frac{e^{2 \gamma k}}{2 k^{2}}(t-k)^{2}+\frac{\gamma}{2} e^{2 \gamma k}(t-k)+\frac{e^{2 \gamma k}}{4}, & t \geq k .\end{cases}
$$

Since $u \in H_{\text {loc }}^{1}(\Omega)$, it follows that $\tau_{k}(u)$ and $\eta_{k}(u) \in H_{\text {loc }}^{1}(\Omega)$ for any $k \in \mathbb{N}$.

Since $u$ is a weak stable solution, applying (1.4) with the test function $\tau_{k}(u) \phi$ where $\phi \in C_{0}^{1}(\Omega)$, we have

$$
\begin{gathered}
\int_{\Omega}\left[e^{u}+\beta|x|^{\alpha} e^{\beta u}\right] \tau_{k}^{2}(u) \phi^{2} \mathrm{~d} x \leq \int_{\Omega}\left|\nabla\left(\tau_{k}(u) \phi\right)\right|^{2} \mathrm{~d} x \\
=\int_{\Omega}\left|\nabla\left(\tau_{k}(u)\right)\right|^{2} \phi^{2}+\tau_{k}^{2}(u)|\nabla \phi|^{2}-\frac{\tau_{k}^{2}(u)}{2} \Delta\left(\phi^{2}\right) \mathrm{d} x .
\end{gathered}
$$

Since $\phi \in C_{0}^{1}(\Omega)$ and $\tau_{k} \in H_{\mathrm{loc}}^{1}(\Omega)$, the last term is finite in the above inequality, which implies that $e^{u} \tau_{k}^{2}(u)$ and $|x|^{\alpha} e^{\beta u} \tau_{k}^{2}(u) \in L_{\mathrm{loc}}^{1}(\Omega)$.

Moreover, using the fact that $\eta_{k}^{\prime}=\left(\tau_{k}^{\prime}\right)^{2}, \eta_{k}=\xi_{k}^{\prime}$,

$$
\begin{aligned}
\int_{\Omega}\left|\nabla\left(\tau_{k}(u)\right)\right|^{2} \phi^{2} \mathrm{~d} x & =\int_{\Omega}\left|\tau_{k}^{\prime}(u) \nabla u\right|^{2} \phi^{2} \mathrm{~d} x=\int_{\Omega} \nabla \eta_{k}(u) \cdot \nabla u \phi^{2} \mathrm{~d} x \\
& =\int_{\Omega} \nabla u \nabla\left(\eta_{k}(u) \phi^{2}\right) \mathrm{d} x-\int_{\Omega} \eta_{k}(u) \nabla u \nabla \phi^{2} \mathrm{~d} x \\
& =\int_{\Omega}\left[e^{u}+|x|^{\alpha} e^{\beta u}\right] \eta_{k}(u) \phi^{2} \mathrm{~d} x+\int_{\Omega} \xi_{k}(u) \Delta\left(\phi^{2}\right) .
\end{aligned}
$$

By the definition of $\xi_{k}$ and $\eta_{k}$, one can easily check that

$$
\eta_{k}(t) \leq\left(\frac{\gamma}{2}+\frac{1}{4 k}\right) \tau_{k}^{2}(t) .
$$

Combining (2.3), (2.4) and (2.5),

$$
\begin{aligned}
& \left(1-\frac{\gamma}{2}-\frac{1}{4 k}\right) \int_{\Omega} e^{u} \tau_{k}^{2}(u) \phi^{2} \mathrm{~d} x+\left(\beta-\frac{\gamma}{2}-\frac{1}{4 k}\right) \int_{\Omega}|x|^{\alpha} e^{\beta u} \tau_{k}^{2}(u) \phi^{2} \mathrm{~d} x \\
& \quad \leq \int_{\Omega}\left[\xi_{k}(u)-\frac{1}{2} \tau_{k}^{2}(u)\right] \Delta\left(\phi^{2}\right) \mathrm{d} x+\int_{\Omega} \tau_{k}^{2}(u)|\nabla \phi|^{2} \mathrm{~d} x .
\end{aligned}
$$


Now set $\phi=\psi^{m}$ with $\psi \in C_{0}^{\infty}(\Omega)$ and $\|\psi\|_{\infty} \leq 1$. Using the fact that $\tau_{k}(u) \leq e^{\gamma u}$ and Hölder inequality, one has

$$
\begin{aligned}
& \int_{\Omega} \tau_{k}^{2}(u)|\nabla \phi|^{2} \mathrm{~d} x=m^{2} \int_{\Omega} \tau_{k}^{2}(u) \psi^{2(m-1)}|\nabla \psi|^{2} \mathrm{~d} x \\
& \quad \leq C \int_{\Omega}\left[e^{\beta u} \tau_{k}^{2}(u)\right]^{\frac{2 \gamma}{\beta+2 \gamma}} \psi^{2(m-1)}|\nabla \psi|^{2} \mathrm{~d} x \\
& \quad \leq C\left(\int_{\Omega}|x|^{\alpha} e^{\beta u} \tau_{k}^{2}(u) \psi^{\frac{(m-1)(\beta+2 \gamma)}{\gamma}} \mathrm{d} x\right)^{\frac{2 \gamma}{\beta+2 \gamma}}\left(\int_{\Omega}|x|^{-\frac{2 \alpha \gamma}{\beta}}|\nabla \psi|^{\frac{2(\beta+2 \gamma)}{\beta}} \mathrm{d} x\right)^{\frac{\beta}{\beta+2 \gamma}} .
\end{aligned}
$$

Choose $m \geq 1+\frac{4}{\max \{1, \beta\}}$ which implies that $\frac{(m-1)(\beta+2 \gamma)}{\gamma} \geq 2 m$ for any $0<\gamma<\min \{2,2 \beta\}$.

Together with $|\psi| \leq 1$, we get $\psi^{\frac{(m-1)(\beta+2 \gamma)}{\gamma}} \leq \psi^{2 m}$ and

$$
\int_{\Omega} \tau_{k}^{2}(u)|\nabla \phi|^{2} \mathrm{~d} x \leq C\left(\int_{\Omega}|x|^{\alpha} e^{\beta u} \tau_{k}^{2}(u) \psi^{2 m} \mathrm{~d} x\right)^{\frac{2 \gamma}{\beta+2 \gamma}}\left(\int_{\Omega}|x|^{-\frac{2 \alpha \gamma}{\beta}}|\nabla \psi|^{\frac{2(\beta+2 \gamma)}{\beta}} \mathrm{d} x\right)^{\frac{\beta}{\beta+2 \gamma}} .
$$

Furthermore, there exists $C>0$ independent of $k$ such that (see [28])

$$
\left|\xi_{k}(u)-\frac{\tau_{k}^{2}(u)}{2}\right| \leq C\left[e^{\beta u} \tau_{k}^{2}(u)\right]^{\frac{2 \gamma}{\beta+2 \gamma}} .
$$

Direct calculation shows that $\Delta\left(\phi^{2}\right)=2 m \psi^{2 m-1} \Delta \psi+2 m(2 m-1) \psi^{2 m-2}|\nabla \psi|^{2}$, applying Hölder inequality again,

$$
\begin{aligned}
& \int_{\Omega}\left[\xi_{k}(u)-\frac{\tau_{k}^{2}(u)}{2}\right] \Delta\left(\phi^{2}\right) \mathrm{d} x \\
& \quad \leq C \int_{\Omega}\left[e^{\beta u} \tau_{k}^{2}(u)\right]^{\frac{2 \gamma}{\beta+2 \gamma}}\left[\psi^{2 m-1}|\Delta \psi|+\psi^{2 m-2}|\nabla \psi|^{2}\right] \mathrm{d} x \\
& \quad \leq C\left(\int_{\Omega}|x|^{\alpha} e^{\beta u} \tau_{k}^{2}(u) \psi^{2 m} \mathrm{~d} x\right)^{\frac{2 \gamma}{\beta+2 \gamma}}\left(\int_{\Omega}|x|^{-\frac{2 \alpha \gamma}{\beta}}\left(|\nabla \psi|^{2}+|\psi||\Delta \psi|\right)^{\frac{\beta+2 \gamma}{\beta}} \mathrm{d} x\right)^{\frac{\beta}{\beta+2 \gamma}} .
\end{aligned}
$$

Combining (2.6), (2.8), (2.9),

$$
\begin{aligned}
& \left(1-\frac{\gamma}{2}-\frac{1}{4 k}\right) \int_{\Omega} e^{u} \tau_{k}^{2}(u) \phi^{2} \mathrm{~d} x+\left(\beta-\frac{\gamma}{2}-\frac{1}{4 k}\right) \int_{\Omega}|x|^{\alpha} e^{\beta u} \tau_{k}^{2}(u) \phi^{2} \mathrm{~d} x \\
& \leq C\left(\int_{\Omega}|x|^{\alpha} e^{\beta u} \tau_{k}^{2}(u) \psi^{2 m} \mathrm{~d} x\right)^{\frac{2 \gamma}{\beta+2 \gamma}}\left(\int_{\Omega}|x|^{-\frac{2 \alpha \gamma}{\beta}}\left(|\nabla \psi|^{2}+|\psi||\Delta \psi|\right)^{\frac{\beta+2 \gamma}{\beta}} \mathrm{d} x\right)^{\frac{\beta}{\beta+2 \gamma}} .
\end{aligned}
$$

If $1-\frac{\gamma}{2}-\frac{1}{4 k}>\delta>0$ and $\beta-\frac{\gamma}{2}-\frac{1}{4 k}>\delta>0$ for some $\delta>0$, which is independent of large $k$, then there exists $C>0$ such that

$$
\int_{\Omega}\left[e^{u}+|x|^{\alpha} e^{\beta u}\right] \tau_{k}^{2}(u) \psi^{2 m} \mathrm{~d} x \leq C \int_{\Omega}|x|^{-\frac{2 \alpha \gamma}{\beta}}\left(|\nabla \psi|^{2}+|\psi||\Delta \psi|\right)^{\frac{\beta+2 \gamma}{\beta}} \mathrm{d} x .
$$

Fixing $\gamma \in(0, \min \{2,2 \beta\})$ and let $k \rightarrow \infty$,

$$
\int_{\Omega}\left[e^{(2 \gamma+1) u}+|x|^{a} e^{(\beta+2 \gamma) u}\right] \psi^{2 m} \mathrm{~d} x \leq C \int_{\Omega}|x|^{-\frac{2 \alpha \gamma}{\beta}}\left(|\nabla \psi|^{2}+|\psi||\Delta \psi|\right)^{\frac{\beta+2 \gamma}{\beta}} \mathrm{d} x .
$$

The proof is completed. 


\subsection{Nonexistence of stable solutions}

We prove here Theorem 1.1. Suppose $u$ is a weak and stable solution to (1.1) with $\Omega=\mathbb{R}^{N}$ and $2 \leq N<2+\frac{4(\alpha+2)}{\max \{1, \beta\}}$. Fix $m \geq 1+\frac{4}{\max \{1, \beta\}}$, and choose $\gamma \in(0, \min \{2,2 \beta\})$ such that $N-\frac{2 \alpha \gamma}{\beta}-\frac{2(\beta+2 \gamma)}{\beta}<0$.

Let $\phi \in C_{0}^{\infty}\left(\mathbb{R}^{N}\right)$ be a cutoff function such that $\phi(x)=1$ for $|x| \leq 1$ and $\phi(x)=0$ for $|x| \geq 2$. Applying Proposition 2.1 with $\psi=\phi_{R}(x)$ where $\phi_{R}(x)=\phi\left(\frac{x}{R}\right)$ and $R>0$,

$$
\begin{aligned}
\int_{B(0, R)}\left[e^{(2 \gamma+1) u}+|x|^{\alpha} e^{(\beta+2 \gamma) u}\right] \mathrm{d} x & \leq \int_{\mathbb{R}^{N}}\left[e^{(2 \gamma+1) u}+|x|^{\alpha} e^{(\beta+2 \gamma) u}\right] \psi^{2 m} \mathrm{~d} x \\
& \leq C \int_{\mathbb{R}^{N}}|x|^{-\frac{2 \alpha \gamma}{\beta}}\left(|\nabla \psi|^{2}+|\psi||\Delta \psi|\right)^{\frac{\beta+2 \gamma}{\beta}} \mathrm{d} x \\
& \leq C R^{N-\frac{2 \alpha \gamma}{\beta}-\frac{2(\beta+2 \gamma)}{\beta}} \text { for } \forall R>0 .
\end{aligned}
$$

Letting $R \rightarrow \infty$, one has

$$
\int_{\mathbb{R}^{N}}\left[e^{(2 \gamma+1) u}+|x|^{\alpha} e^{(\beta+2 \gamma) u}\right] \mathrm{d} x=0,
$$

which is impossible.

\subsection{Nonexistence for finite Morse index solutions}

We prove here Theorem 1.2. Suppose $u$ is a weak solution of (1.1) which has finite Morse index. There exists a compact set $\mathcal{K}$ such that $u$ is stable in $\mathbb{R}^{N} \backslash \mathcal{K}$. There exists $R_{0}>0$ such that $\mathcal{K} \subset B\left(0, R_{0}\right)$. Therefore, we can apply Proposition 2.1 with $\Omega=\mathbb{R}^{N} \backslash B\left(0, R_{0}\right)$.

First we have the following estimates:

Lemma 2.2 For any $\gamma \in(0, \min \{2,2 \beta\})$ and any $R>2 R_{0}$, there holds

$$
\int_{2 R_{0}<|x|<R} e^{(2 \gamma+1) u}+|x|^{\alpha} e^{(\beta+2 \gamma) u} d x \leq A+B R^{N-\frac{2 \alpha \gamma}{\beta}-\frac{2(\beta+2 \gamma)}{\beta}},
$$

where $A, B>0$ are constants independent of $R$. Moreover, for $B(y, 2 r) \subset \mathbb{R}^{N} \backslash \overline{B\left(0, R_{0}\right)}$, there holds

$$
\int_{B(y, r)} e^{(2 \gamma+1) u}+|x|^{\alpha} e^{(\beta+2 \gamma) u} d x \leq C r^{N-\frac{2 \alpha \gamma}{\beta}-\frac{2(\beta+2 \gamma)}{\beta}}
$$

for some $C>0$ independent of $r>0$.

Proof Let $R>2 R_{0}$. Define $\psi_{R}=\phi_{R}-\phi_{R_{0}}$ with $\phi_{r}$ being defined as before. Notice that $\psi_{R} \in C_{0}^{\infty}\left(\mathbb{R}^{N} \backslash \mathcal{K}\right), 0 \leq \psi_{R} \leq 1$ and it is a fixed function $\eta_{0}$ in $B\left(0,2 R_{0}\right)$ which is independent of $R$. Hence, we can apply Proposition 2.1 here,

$$
\begin{aligned}
& \int_{2 R_{0}<|x|<R} e^{(2 \gamma+1) u}+|x|^{\alpha} e^{(\beta+2 \gamma) u} \mathrm{~d} x \\
& \leq \int_{\mathbb{R}^{N} \backslash \mathcal{K}}\left[e^{(2 \gamma+1) u}+|x|^{\alpha} e^{(\beta+2 \gamma) u} \mathrm{~d} x\right] \psi_{R}^{2 m} \mathrm{~d} x \\
& \leq C \int_{\mathbb{R}^{N} \backslash \mathcal{K}}|x|^{-\frac{2 \alpha \gamma}{\beta}}\left(\left|\nabla \psi_{R}\right|^{2}+\left|\psi_{R}\right|\left|\Delta \psi_{R}\right|\right)^{\frac{\beta+2 \gamma}{\beta}} \mathrm{d} x
\end{aligned}
$$




$$
\begin{aligned}
\leq & C \int_{R_{0}<|x|<2 R_{0}}|x|^{-\frac{2 \alpha \gamma}{\beta}}\left(\left|\nabla \eta_{0}\right|^{2}+\left|\eta_{0}\right|\left|\Delta \eta_{0}\right|\right)^{\frac{\beta+2 \gamma}{\beta}} \mathrm{d} x \\
& +C \int_{R<|x|<2 R}|x|^{-\frac{2 \alpha \gamma}{\beta}}\left(\left|\nabla \psi_{R}\right|^{2}+\left|\psi_{R}\right|\left|\Delta \psi_{R}\right|\right)^{\frac{\beta+2 \gamma}{\beta}} \mathrm{d} x \\
\leq & A+B R^{N-\frac{2 \alpha \gamma}{\beta}-\frac{2(\beta+2 \gamma)}{\beta}},
\end{aligned}
$$

where $A, B>0$ are two positive constants depending on $\alpha, \beta, \gamma, R_{0}$ but independent of $R$. Thus we have

$$
\int_{2 R_{0}<|x|<R} e^{(2 \gamma+1) u}+|x|^{\alpha} e^{(\beta+2 \gamma) u} \mathrm{~d} x \leq A+B R^{N-\frac{2 \alpha \gamma}{\beta}-\frac{2(\beta+2 \gamma)}{\beta}} .
$$

Similarly, for $B(y, 2 r) \subset \mathbb{R}^{N} \backslash \overline{B\left(0, R_{0}\right)}$, we can apply Proposition 2.1 with the test function $\phi_{r}(x-y)$, one can obtain the following:

$$
\int_{B(y, r)} e^{(2 \gamma+1) u}+|x|^{\alpha} e^{(\beta+2 \gamma) u} \mathrm{~d} x \leq C r^{N-\frac{2 \alpha \gamma}{\beta}-\frac{2(\beta+2 \gamma)}{\beta}}
$$

for some $C>0$ independent of $r>0$. We are done.

\section{Lemma 2.3}

$$
\lim _{|x| \rightarrow \infty}|x|^{\frac{\alpha+2}{\beta}} e^{u(x)}=0 .
$$

Proof Let $|y|>4 R_{0}$ such that $B(y, 2 R) \subset \mathbb{R}^{N} \backslash \overline{B\left(0, R_{0}\right)}$. We denote $f(x, u)=e^{u}+$ $|x|^{\alpha} e^{\beta u}$. If $\frac{N}{2}<1+\frac{2 \gamma}{\max \{1, \beta\}}$, i.e., $N<2+\frac{4 \gamma}{\max \{1, \beta\}}\left(N<2+8 \min \left\{\beta, \frac{1}{\beta}\right\}\right)$, then by Hölder inequality,

$$
\begin{aligned}
& \int_{B(y, R)} f(x, u)^{\theta} \mathrm{d} x \leq C_{\theta} \int_{B(y, R)} e^{\theta u}+|x|^{\alpha \theta} e^{\beta \theta u} \mathrm{~d} x \\
& \leq C\left(\int_{B(y, R)} e^{(2 \gamma+1) u} \mathrm{~d} x\right)^{\frac{\theta}{2 \gamma+1}}\left(\int_{B(y, R)} 1 \mathrm{~d} x\right)^{\frac{2 \gamma+1-\theta}{2 \gamma+1}} \\
& \quad+C\left(\int_{B(y, R)}|x|^{\alpha} e^{(\beta+2 \gamma) u} \mathrm{~d} x\right)^{\frac{\beta \theta}{\beta+2 \gamma}}\left(\int_{B(y, R)}^{\left.|x|^{\frac{2 \alpha \gamma \theta}{\beta+2 \gamma-\beta \theta}} \mathrm{d} x\right)^{\frac{\beta+2 \gamma-\beta \theta}{\beta+2 \gamma}}}\right. \\
& \leq C\left(R^{\left(N-\frac{2 \alpha \gamma}{\beta}-\frac{2(\beta+2 \gamma)}{\beta}\right) \frac{\theta}{2 \gamma+1}+\frac{N(2 \gamma+1-\theta)}{2 \gamma+1}}+R^{\left(N-\frac{2 \alpha \gamma}{\beta}-\frac{2(\beta+2 \gamma)}{\beta}\right) \frac{\beta \theta}{\beta+2 \gamma}+\left(N+\frac{2 \alpha \gamma \theta}{\beta+2 \gamma-\beta \theta}\right) \frac{\beta+2 \gamma-\beta \theta}{\beta+2 \gamma}}\right) \\
& \leq C\left(R^{N-2 \theta \frac{2 \gamma+\alpha \gamma+\beta}{(2 \gamma+1) \beta}}+R^{N-2 \theta}\right) \leq C R^{N-2 \theta},
\end{aligned}
$$

when $\frac{\alpha+2}{\beta} \geq 2$, where we used (2.15). So we have

$$
\int_{B(y, R)} f(x, u)^{\theta} \mathrm{d} x \leq C R^{N-2 \theta} \quad \text { for } \quad|y|>4 R_{0}, \quad R=\frac{|y|}{4} .
$$

In order to prove Lemma 2.3, we need the following result of Serrin [25].

Lemma 2.4 Let $\theta=\frac{N}{2-\epsilon_{0}}, \epsilon_{0} \in(0,2), q \in(1, \infty]$ and $\delta>0$. For any weak solution of $-\Delta u=a(x) u$ in $B(y, 2 R)$, if

$$
R^{\epsilon_{0}}\|a(x)\|_{L^{\theta}(B(y, 2 R))} \leq \delta,
$$


there holds,

$$
\|u\|_{L^{\infty}}(B(y, R)) \leq C R^{-\frac{N}{q}}\|u\|_{L^{q}(B(y, 2 R))}
$$

where $C$ is a constant depending on $N, q, \theta$ and $\delta$. Moreover, the above estimate also holds for any nonnegative weak solution of $-\Delta u \leq a(x) u$ in $B(y, 2 R)$ provided (2.22) holds.

Let us continue the proof of Lemma 2.3. Set

$$
\beta_{1}=\frac{(N-2) \beta}{2(\alpha+2)}, \quad \lambda=\frac{\beta+2 \beta_{1}}{2}>0, \quad w=e^{\lambda u} .
$$

In this case, $\beta_{1} \in(0, \min \{2,2 \beta\})$ since $N \in\left(2,2+\frac{4(\alpha+2)}{\max \{1, \beta\}}\right)$ and $N-\frac{2 \alpha \beta_{1}}{\beta}-\frac{2\left(\beta+2 \beta_{1}\right)}{\beta}=0$. Letting $R \rightarrow 0$ in (2.14), we have

$$
\int_{|x|>2 R_{0}}|x|^{\alpha} e^{\beta+2 \beta_{1}} \mathrm{~d} x=\int_{|x|>2 R_{0}}|x|^{\alpha} w^{2} \mathrm{~d} x \leq C<\infty,
$$

with some generic constant $C$ which is independent of $R_{0}$ and $R$. We also have that

$$
\Delta w+\lambda\left(e^{u}+|x|^{\alpha} e^{\beta u}\right) w=\lambda^{2} e^{\lambda u}|\nabla u|^{2} \geq 0 .
$$

So Lemma 2.4 can be applied for this equation. Applying this lemma with $a(x)=\lambda\left(e^{u}+\right.$ $\left.|x|^{\alpha} e^{\beta u}\right), q=2$, one can check that by (2.21)

$$
R^{\epsilon_{0}}\|a(x)\|_{L^{\theta}(B(y, 2 R))} \leq C R^{\epsilon_{0}+\frac{N-2 \theta}{\theta}} \leq C,
$$

for all $|y|>8 R_{0}$ and $R=\frac{|y|}{4}$.

Applying Lemma 2.4 with $\delta=C$ in the above estimate, one has

$$
w(y) \leq C R^{-\frac{N}{2}}\|w\|_{L^{2}(B(y, 2 R))} \leq C R^{-\frac{N}{2}-\frac{\alpha}{2}}\left\||x|^{\frac{\alpha}{2}} w\right\|_{L^{2}(B(y, 2 R))}=o\left(R^{-\frac{N+\alpha}{2}}\right)
$$

as $|y| \rightarrow \infty$ by (2.25). By the definition of $w$,

$$
e^{u(y)}=w^{\frac{1}{\lambda}}=o\left(R^{-\frac{N+\alpha}{2 \lambda}}\right)=o\left(R^{-\frac{\alpha+2}{\beta}}\right)=o\left(|y|^{-\frac{\alpha+2}{\beta}}\right) \text { as }|y| \rightarrow \infty .
$$

We are done.

In the end of this section, let us finish the proof of Theorem 1.2.

Proof of Theorem 1.2 To finish the proof, consider $v$, the average of $u$ over sphere. First fix $M>0$ such that $\frac{\alpha+2}{\beta}-\frac{2}{(N-2) M}>0$. By (2.19), there exists $R_{M}>0$ such that

$$
-\Delta v=\overline{e^{u}}+\overline{|x|^{\alpha} e^{\beta u}} \leq \frac{1}{2 M r^{\frac{\alpha+2}{\beta}}}+\frac{1}{2 M r^{2}} \leq \frac{1}{M r^{2}}, \quad \forall r \geq R_{M},
$$

where we used $\frac{\alpha+2}{\beta} \geq 2$.

Integrating from $R_{M}$ to $r$, we have

$$
v^{\prime}(r) \geq-\frac{C}{r^{N-1}}-\frac{1}{(N-2) M r}, \quad \forall r \geq R_{M} .
$$

As $N>2$, there exists $R^{\prime}>R_{M}$ such that

$$
v^{\prime}(r) \geq-\frac{2}{(N-2) M r}, \quad \forall r \geq R^{\prime} .
$$

Integrating from $R^{\prime}$ to $r$,

$$
r^{\frac{\alpha+2}{\beta}} e^{v(r)} \geq C r^{\frac{\alpha+2}{\beta}-\frac{2}{(N-2) M}},
$$


which implies

$$
\sup _{|x|=r}\left(r^{\frac{\alpha+2}{\beta}} e^{u(x)}\right) \geq r^{\frac{\alpha+2}{\beta}} e^{v(r)} \geq C r^{\frac{\alpha+2}{\beta}-\frac{2}{(N-2) M}} \rightarrow \infty
$$

and it contradicts with (2.19). Thus we finish the proof.

Acknowledgements The research of the first author is supported by NSFC Nos.11801421 and 11631011. The research of the second author is supported by NSFC Nos.11801550 and 11871470.

\section{References}

1. Ambjorn, J., Olesen, P.: Anti-screening of large magnetic fields by vector bosons. Phys. Lett. B 214, 565-569 (1988)

2. Bartolucci, D., Castorina, D.: Self-gravitating cosmic strings and the Alexandrov's inequality for Liouville-type equations. Commun. Contemp. Math. 18(4), 26 (2016)

3. Chae, D.: Existence of a semilinear elliptic system with exponential nonlinearities. Discrete Contin. Dyn. Syst. 18, 709-718 (2007)

4. Chae, D.: Existence of multistring solutions of a selfgravitating massive Wboson. Lett. Math. Phys. 73, 123-134 (2005)

5. Chen, R.M., Guo, Y., Spirn, D.: Asymptotic behaviour and symmetry of condensate solutions in electroweak theory. J. Anal. Math. 117, 47-85 (2012)

6. Dancer, E.N.: Finite Morse index solutions of exponential problems. Ann. Inst. H. Poincaré Anal Non Linéaire 25, 173-179 (2008)

7. Dancer, E.N., Farina, A.: On the classification of solutions of $-\Delta u=e^{u}$ on $\mathbb{R}^{N}$ : stability outside a compact set and applications. Proc. Am. Math. Soc. 137(4), 1333-1338 (2009)

8. Dancer, E.N., Du, Y., Guo, Z.M.: Finite Morse index solutions of an elliptic equation with supercritical exponent. J. Differ. Equ. 250, 3281-3310 (2011)

9. Dávila, J., Dupaigne, L., Farina, A.: Partial regularity of finite Morse index solutions to the Lane-Emden equation. J. Funct. Anal. 261(1), 218-232 (2011)

10. Dávila, J., Ye, D.: On finite Morse index solutions of two equations with negative exponent. Proc. R. Soc. Edinb. Sect. A 143(1), 121-128 (2013). (preprint)

11. Dávila, J., Dupaigne, L., Wang, K.L., Wei, J.C.: A monotonicity formula and a Liouville-type theorem for a fourth order supercritical problem. Adv. Math. 258, 240-285 (2014)

12. De Marchis, F., Ricciardi, T.: Existence results for turbulent flows with arbitrary intensities. Nonlinear Anal. Real. 38, 222-244 (2017)

13. Dupaigne, L., Ghergu, M., Goubet, O., Warnault, G.: The Gel'fand problem for the biharmonic operator. Arch. Ration. Mech. Anal. 208, 725-752 (2013)

14. Farina, A.: On the classification of solutions of the Lane-Emden equation on unbounded domains of $\mathbb{R}^{N}$. J. Math. Pures Appl. 87, 537-561 (2007)

15. Gui, C., Jevnikar, A., Moradifam, A.: Symmetry and uniqueness of solutions to some Liouville-type equations and systems. Comm. PDEs 43(3), 428-447 (2018)

16. Hajlaoui, H., Harrabi, A.A., Ye, D.: On stable solutions of biharmonic problem with polynomial growth. Pac. J. Math. 270(1), 79-93 (2014)

17. Jevnikar, A., Yang, W.: A mean field equation involving positive supported probability measures: blow-up phenomena and variational aspects. Proc. R. Soc. Edinb. A (2018). https://doi.org/10.1017/prm.2018.30

18. Ohtsuka, H., Ricciardi, T., Suzuki, T.: Blow-up analysis for an elliptic equation describing stationary vortex flows with variable intensities in 2D-turbulence. J. Differ. Equ. 249, 1436-1465 (2010)

19. Onsager, L.: Statistical hydrodynamics. Nuovo Cimento Suppl. 6, 279-287 (1949)

20. Poliakovsky, A., Tarantello, G.: On a planar Liouville-type problem in the study of self-gravitating strings. J. Differ. Equ. 252(5), 3668-3693 (2012)

21. Poliakovsky, A., Tarantello, G.: On Singular Liouville Systems, Analysis and Topology in Nonlinear Differential Equations. PNDLE 85 Birkhauser, Cham (2014)

22. Poliakovsky, A., Tarantello, G.: On non-topological solutions for planar Liouville systems of Toda-type. Commun. Math. Phys. 347(1), 223-270 (2016)

23. Ricciardi, T., Zecca, G.: Blow-up analysis for some mean field equations involving probability measures from statistical hydrodynamics. Differ. Integral Equ. 25(3-4), 201-222 (2012) 
24. Sawada, K., Suzuki, T.: Derivation of the equilibrium mean field equations of point vortex and vortex filament system. Theor. Appl. Mech. Jpn. 56, 285-290 (2008)

25. Serrin, J.: Local behavior of solutions of quasi-linear equations. Acta Math. 111, 247-302 (1964)

26. Tarantello, G.: Blow-up analysis for a cosmic strings equation. J. Funct. Anal. 272(1), 255-338 (2017)

27. Tarantello, G.: Analytical issues in the construction of self-dual Chern-Simons vortices. Milan J. Math. 84(2), 269-298 (2016)

28. Wang, C., Ye, D.: Some Liouville theorems for Hénon type elliptic equations. J. Funct. Anal. 262(4), $1705-1727(2012)$

29. Wang, K.L.: Stable solutions for Toda system. (preprint)

30. Wei, J.C., Xu, X., Yang, W.: On the classification of stable solutions to biharmonic problems in large dimensions. Pac. J. Math. 263(2), 495-512 (2013)

31. Wei, J.C., Ye, D.: Liouville theorems for finite Morse index solutions of biharmonic problem. Math. Ann. 356(4), 1599-1612 (2013)

32. Yang, Y.: Solitons in Field Theory and Nonlinear Analysis. Springer, New York (2001)

33. Yang, Y.: Self duality of the gauge field equations and the cosmological constant. Commun. Math. Phys. 162, 481-498 (1994)

Publisher's Note Springer Nature remains neutral with regard to jurisdictional claims in published maps and institutional affiliations. 